\title{
On the impact of substrate electron injection on dynamic Ron in GaN- on-Si HEMTs
}

\author{
Dario Pagnano ${ }^{\mathrm{a}, *}$, Giorgia Longobardi ${ }^{\mathrm{a}}$, Florin Udrea ${ }^{\mathrm{a},}$ Jinming Sun $^{\mathrm{b}}$, Mohamed \\ Imam $^{\mathrm{b}}$, Reenu Garg ${ }^{\mathrm{b}}$, Hyeongnam Kim ${ }^{\mathrm{b}}$, Alain Charles ${ }^{\mathrm{b}}$
}

${ }^{a}$ University of Cambridge, Engineering Department, Cambridge, U.K.

${ }^{\mathrm{b}}$ Infineon Technologies Americas Corp, El Segundo, CA 90245, USA

\begin{abstract}
The impact of electron injection from the substrate on the dynamic Ron of GaN-on-Si High Electron Mobility Transistors (HEMTs) has been investigated by means of back-bias transient and vertical leakage measurements and TCAD simulations. A strong correlation between electrons injected from the substrate and on-state drain current transients is demonstrated. Moreover, the contribution of the electron-type traps in the buffer layer as opposed to the usually studied hole-like traps to the dynamic Ron is discussed. In particular, the impact of electron-like traps for different levels of substrate leakage current is studied. A TCAD model has been developed and calibrated by taking into account both off-state vertical leakage and on-state drain current transient experimental results. The proposed charge dynamic has also been assessed against state-of-the-art theories. This analysis contributes to a deeper understanding of the complex scenario of different types of traps in the buffer layer of GaN-on-Si devices and highlights the impact that trap-states can have on the on-state and off-state currents.
\end{abstract}

\section{Introduction}

When compared to silicon solutions for power applications, GaN-based HEMTs have smaller form factor, offer lower on-state losses and are able to switch at higher frequencies. This makes them promising candidates for high-efficient power conversion systems [1]. Despite the great progress both at material and device design level achieved in the last decades, these devices still suffer from reliability issues such as dynamic Ron, a decrease in the drain current after high-voltage stress is applied. The impact of carbon doping on the dynamic characteristics of GaN-on-Si is highly debated in this respect. It has been shown that carbon, usually introduced to improve the vertical blocking capability of the device, may introduce a deep acceptor in the bottom half of the bandgap [2] and holes emitted from these deep levels during off-state operation might create a negatively charged area [3]. This negative charge would partially deplete the two-dimensional electron gas (2DEG) after the off-state stress is removed. Other studies $[4,5]$ discuss a correlation between the vertical leakage and dynamic Ron attributing the reduced 2DEG concentration to the negative charge of the electrons injected from the substrate to trap states in the buffer.

This paper aims at clarifying the correlation between electron injection from the substrate and buffer trapping dynamics coupling these two phenomena, rather than considering them in isolation. This is obtained by experimental results of back-bias measurements at different temperatures and vertical leakage current. A TCAD model which accounts for the electron injection is developed to support the theory proposed.

\section{Experimental results}

The devices under test are packaged TLM structures grown on p-type silicon substrates. Details of the buffer layer and carbon doping are reported in [6]. In order to characterise buffer traps, back-gating measurements and vertical leakage measurements are performed. For the 
back-gating measurements, the on-state current is monitored for $10^{4} \mathrm{~s}$ while a bias of $-100 \mathrm{~V}$ is applied on the substrate. The source contact is grounded and the drain is biased at $20 \mathrm{mV}$. The low drain voltage assures no self-heating effects. Measurements are taken at $\mathrm{T}=25$, $75,100,125^{\circ} \mathrm{C}$ with the aid of an environmental chamber. Vertical off-state currents are obtained by biasing to ground source and drain contact and applying a negative bias to the substrate.

The experimental results in Fig. 1 show an evident decrease of the drain current over time after the stress is applied. This process is accelerated by temperature, as also reported elsewhere [7].

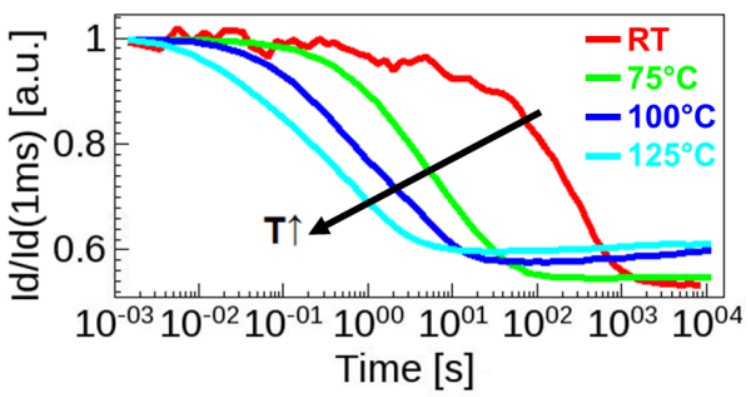

Fig. 1. Measured drain current transient response during Vsub $=|100 \mathrm{~V}|$ voltage stress for $\mathrm{T}=\mathrm{RT}, 75,100,125^{\circ} \mathrm{C}$. The current is normalized to the value at the beginning of the stress $(\mathrm{t}=1 \mathrm{~ms})$.

The vertical leakage at room temperature of the device under test has been measured at the substrate terminal, when its bias was ramped from $0 \mathrm{~V}$ to $-400 \mathrm{~V}$ with a ramp rate of $0.1 \mathrm{~V} / \mathrm{s}$. Fig. 2 shows the vertical leakage as a function of the absolute value of the substrate voltage. For the analysis that will follow in this work, it is important to note that the current at $\mathrm{V}_{\text {sub }}=|100 \mathrm{~V}|$ is approximately $3 \mathrm{nA} / \mathrm{cm}^{2}$ and increases exponentially with the voltage reaching $8 \times 10^{-5} \mathrm{~A} / \mathrm{cm}^{2}$ at $\mathrm{V}_{\mathrm{sub}}=|400 \mathrm{~V}|$.

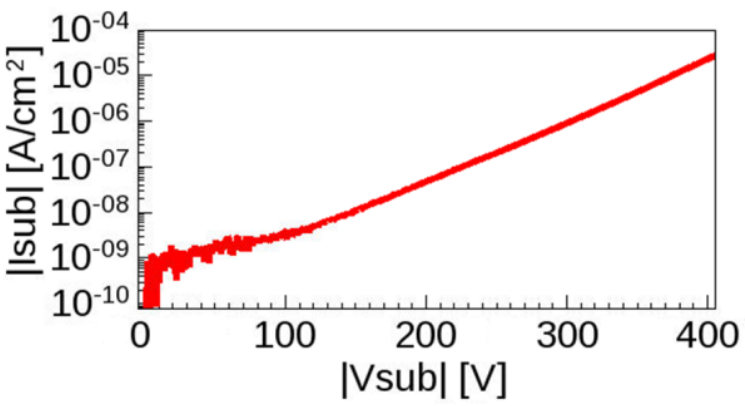

Fig. 2. Measured vertical leakage current Isub as a function of the absolute value of the substrate voltage.

\section{TCAD approach and discussion}

In our TCAD model three important steps were taken to model the electron injection from the substrate and correlate it to the decrease in current shown in Fig.1 and thus to the dynamic Ron: (i) modelling the buffer layer as unique GaN layer, (ii) modelling the region below the $\mathrm{GaN}$ uniform buffer as an equivalent electron injector, (iii) analysing the impact of carbon as an electron-trap. The reasons behind this approach are here explained. Firstly, having a single-material layer as a buffer is a common approach to simplify the complex structure of it $[8,9]$. In particular, we considered as a buffer a single $\mathrm{GaN}$ layer in order to neglect the impact of the energy barriers given by bandgap discontinuities on the carrier transport. These barriers might be not present in reality or their levels may be lower than theoretically predicted values, due to non-ideal interfaces and dislocations. In [6] we have carefully considered the impact of such barriers on the vertical leakage current. In this work, our objective is to clarify whether the electrons injected from the substrate could explain partially or entirely the decrease in the 2DEG charge during the on-state, without focusing on the particular source of carrier and conduction mechanisms.

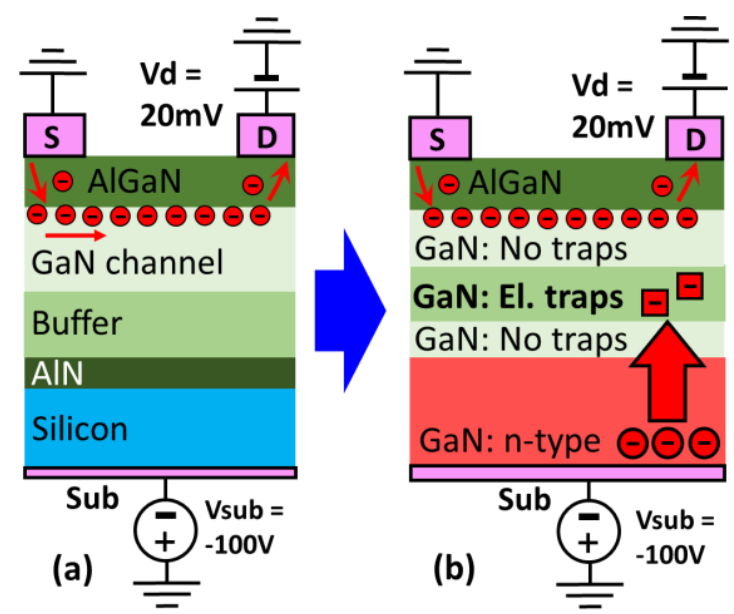

Fig.3: Modelling of the real structure as a simplified buffer and equivalent injector. (a) schematic cross section of the devices under test. (b) illustration of the model implemented in TCAD, where the buffer has been replaced by a single GaN layer with non-uniform trap distribution and the AlN-silicon system has been replaced by an "equivalent injector", implemented as a GaN layer with fully active ntype doping.

For this reason, we have modelled the nucleation layer and substrate (and the additional 2DEG present at their 
interface), with a single layer of $\mathrm{GaN}$ with active ndoping, which performs the role of an electron injection layer, as schematically illustrated in Fig.3. When the simplified n-type GaN layer is introduced as an equivalent substrate, the source of electron will not introduce any time-dependent effects and the strength of the electron injection can be directly adjusted by varying the n-type doping concentration of the GaN injector layer. The doping of the equivalent injector is varied in our analysis and can be chosen to match the measured leakage currents. Given the fact that vertical leakage currents have been reported in literature with values ranging from $10^{-5}$ to $10^{-10} \mathrm{~A} / \mathrm{cm}^{2}[7,9]$, being able to adjust the model according to the vertical current value is extremely important to guarantee the adaptability of the analysis to other structures for which the leakage current is different than the one measured in our devices.

Lastly, another crucial issue for a complete TCAD model of GaN power devices is the modelling of carbon as a trap. In a recent TCAD study by Chini et al. [7], different energy configurations for the trap were investigated and it was concluded that only a hole-trap would lead to a behaviour coherent with current collapse, while an electron-trap would instead lead to increased currents, after the high electrical field stress. In this study, we focus our attention on the behaviour of electron-traps. In our simulation model we assume a single energy level electron trap uniformly located in a $2 \mu \mathrm{m}$ region $200 \mathrm{~nm}$ below the $2 \mathrm{DEG}$. This region corresponds to the one in our sample doped with high carbon concentration. The energy level and cross section were extracted from Arrhenius plots extrapolated from the measurements in Fig.1, while the concentration was fixed at the value of the carbon doping atomistic concentration, extracted by SIMS measurements.

Fig. 4 shows the substrate current transient response to a voltage step of $\mathrm{V}_{\text {sub }}=|100 \mathrm{~V}|$ as a function of the $\mathrm{n}$ type doping concentration of the equivalent injector layer. It needs to be stressed that the values in Fig.4, which range from $5 \times 10^{1} \mathrm{~cm}^{-3}$ to $5 \times 10^{5} \mathrm{~cm}^{-3}$, are not meant to match the substrate doping of the real sample, but correlate to the amount of charge injected from the substrate, which is much lower than its doping because it is limited by conduction barriers. One can note that a concentration of $5 \times 10^{2} \mathrm{~cm}^{-3}$ results in a current of approximately $3 \mathrm{nA} / \mathrm{cm}^{2}$, which corresponds to the measured vertical leakage at $\mathrm{V}_{\text {sub }}=|100 \mathrm{~V}|$ (Fig.2). It is also worth noting that the substrate current shown in Fig. 4 is constant at the beginning of the transient for all values of the injector doping. This is consistent with our approach of modelling the source of electrons as directly available and therefore independent from any timerelated mechanisms. At the same time, for Time $>10^{2} \mathrm{~s}$, the simulations in Fig.4 show a decrease in current over time for doping concentrations higher than $1 \times 10^{5} \mathrm{~cm}^{-3}$. This is caused by the trapping of negative charge in the buffer opposing further electron injection.

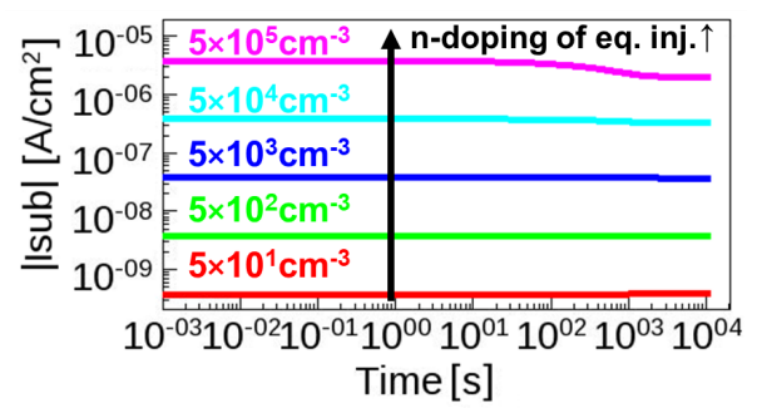

Fig.4: Simulated substrate current as a function of time for different values of the n-type doping of the equivalent injector.

The substrate current values |Isub| corresponding to different doping levels of the equivalent substrate injector have been extracted at the beginning of the transient from Fig.4 and reported in Fig.5. It needs to be stressed that while the substrate doping does not correspond to any fabricated device, the substrate current values do match a range of fabricated devices [7,9].

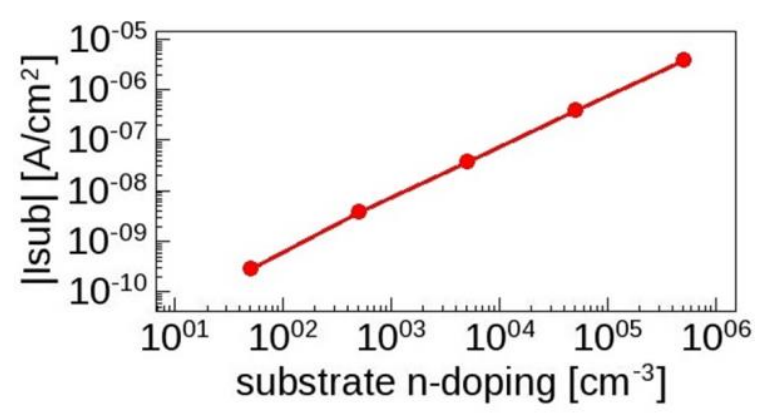

Fig.4: Substrate current |Isub| corresponding to different levels of n-type doping of the equivalent electron injecting substrate.

Based on the information from Fig.5, it is now possible to analyse the impact that a realistic substrate current has on the drain transients and therefore the impact of injected electron trapping on the 2DEG.

Fig.6 shows the drain current transients at different levels of substrate currents corresponding to the different ndoping concentrations as reported in Fig.5. One can note that for low values of vertical leakage, the drain current shows an increase in current that is due to a partial de- 
ionization of the electron-traps. Interestingly an opposite trend occurs for Isub> $4 \times 10^{-8} \mathrm{~A} / \mathrm{cm}^{2}$. As the vertical leakage increases, the drain current transient exhibits a significant decrease in value that can reach up to $15 \%$ for a substrate current of $4 \times 10^{-6} \mathrm{~A} / \mathrm{cm}^{2}$. This percentage corresponds to situations normally observed in literature when performing back bias measurements on $\mathrm{GaN}$ devices.

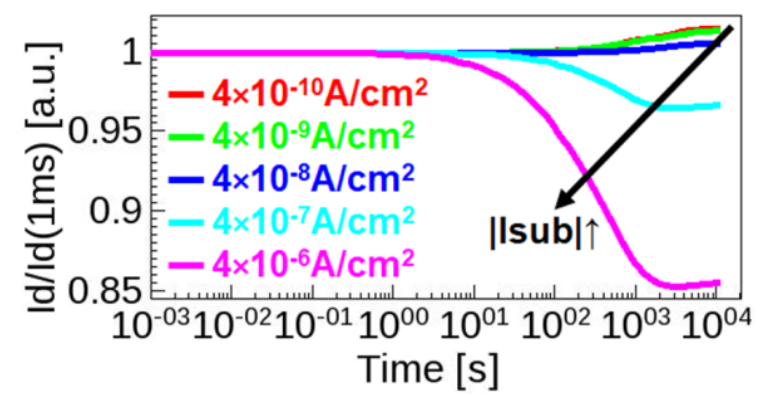

Fig.6: Simulated drain current transient for different substrate leakage values as extracted from the y-axis of Fig.4.

While in this particular set of simulations the electron-traps are assumed to be spatially uniformly distributed within a layer of the device, electron traps may be spatially localized and be present together with hole traps. To have a complete understanding of how each type of traps affect the measurement in Fig. 1, we have substituted the electron-traps with hole-traps. The energy level and cross section are the same as in the previous case, while the concentration is used as a parameter to fit the current decrease observed in Fig.1. Fig. 7 shows the results of this analysis.

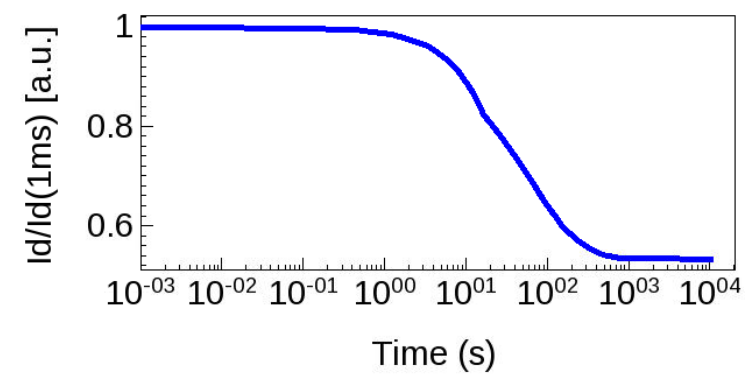

Fig.7: Simulated drain current transient obtained including hole-traps in the model. The trap energy level is $\mathrm{E}_{\mathrm{T}}=\mathrm{E}_{\mathrm{V}}+0.65 \mathrm{eV}$ and the concentration equal to $3 \times 10^{16} \mathrm{~cm}^{-3}$.

The main difference that can be noticed when comparing the case of hole-trap (Fig.7) and electron-trap (Fig.6) is that for the hole-trap case the substrate current does not show any dependence from the injector doping (the transient for different doping levels are superimposed) and a trap concentration much lower than the nominal carbon doping is required to obtain a substantial current decrease. This is consistent with what has been observed in [7].

The temperature dependence of the current transient has been investigated to further clarify the correlation between vertical leakage and electron trap dynamics. Firstly we show the results and discuss the case for an electron-trap distribution, secondly we will analyse the case of a hole-trap distribution and demonstrate that the latter is more suitable for explaining the measured results in Fig.1.

Varying the temperature in the electron-trap model does not lead to a decrease in time constant of the transient, as shown in Fig.8. On the contrary, the drain increases for $\mathrm{T}=450 \mathrm{~K}$.

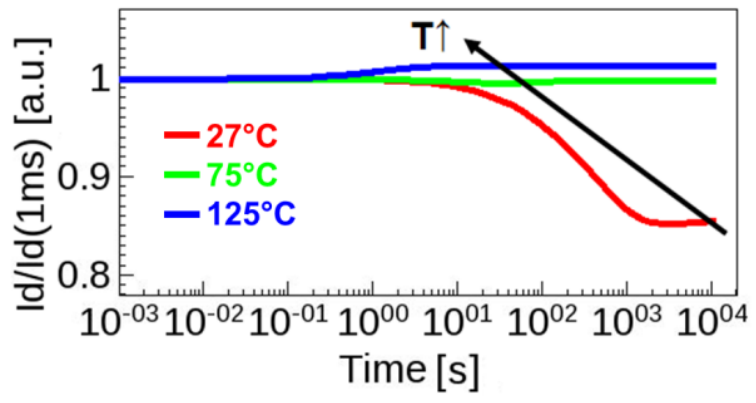

Fig.8: Simulated drain current transient response of the "equivalent injector" and electron-trap model to a $|100 \mathrm{~V}|$ substrate stress for $27^{\circ} \mathrm{C}$, $75^{\circ} \mathrm{C}$ and $125^{\circ} \mathrm{C}$.

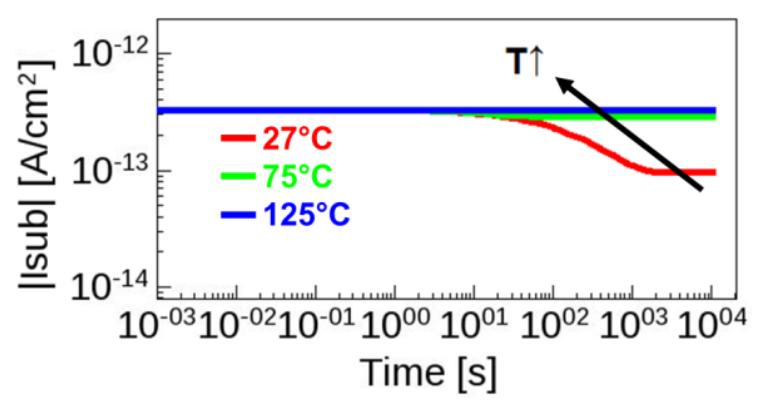

Fig.9: Simulated substrate current transient response of the "equivalent injector" model to a $|100 \mathrm{~V}|$ substrate stress for $\mathrm{T}=27^{\circ} \mathrm{C}, 75^{\circ} \mathrm{C}, 125^{\circ} \mathrm{C}$

The increase in drain current with temperature is due to the fact that the capture process is less effective for higher temperatures and the injected substrate current is constant with temperature. This is shown in Fig.9, where the substrate currents for different temperatures are equal up to 10 s, while the current at $27^{\circ} \mathrm{C}$ for Time $>10$ s shows 
a significant decrease. This is due to the fact that relevant trapping opposes further injection. It is worth mentioning that the temperature dependence of the mobility has not been included in this model.

Fig.10 shows that when the electron-trap is substituted with the hole-trap, the simulations are able to reproduce the measured temperature dependence of the drain current. This is due to the hole emission process (as also discussed in [7]) and has no correlation to the vertical leakage.

The observation that for the electron-trap case the temperature reduces the trapping is a solid logical step to reinforce the correlation between vertical current and a decrease in drain current.

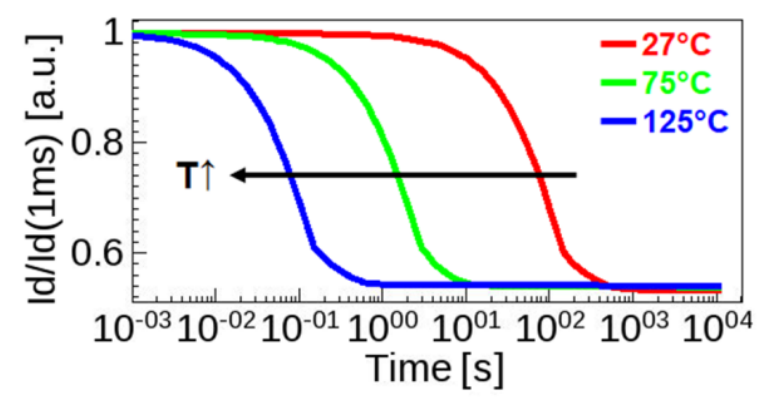

Fig.10: Simulated drain current transient of the "equivalent injector" model for $\mathrm{T}=27^{\circ} \mathrm{C}, 75^{\circ} \mathrm{C}, 125^{\circ} \mathrm{C}$ when the electron-trap is substituted by a hole-trap.

To demonstrate that electron capture can validate the temperature dependence shown in Fig.1, the model is modified to include an AIN layer and silicon substrate below the same buffer. The new cross section considered is shown in Fig. 11(b). The region above the equivalent injector is not modified. In this way, the changes in the dynamics are entirely due to the different injection mechanisms. In the following numerical results concerning the model as in Fig.11(b) only electron traps will be considered, as it has been demonstrated that the hole-trap case is not affected by the injection of electrons.

The physics of the AlN-Si system is complex compared to our previously discussed simplified approach. However, it has been already demonstrated that the barrier at the AlN-Si interface is one of the main parameters controlling the vertical leakage current [6, 8]. Therefore we can consider that the AlN-Si barrier plays an equivalent role to that of the electron injector layer. The temperature dependence of this new model will be however also dependent on the thermionic emission of carriers across the AlN/Si barrier, which we have so far neglected.

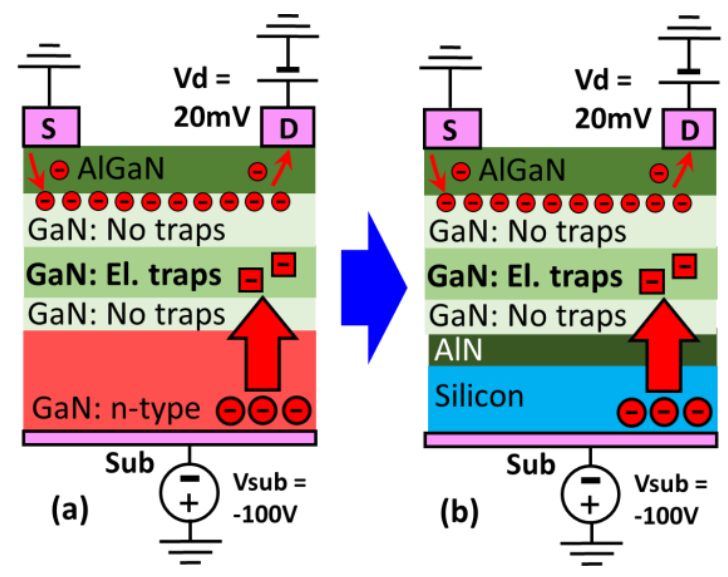

Fig.11: (a) "Equivalent injector" model as introduced in Fig.1. (b) Cross section of the simulated device including the AlN layer and silicon substrate.

Fig.12 shows the drain current transient as a function of temperature for a barrier of $0.6 \mathrm{eV}$, chosen in agreement with $[6,8]$. The temperature dependence is now qualitatively similar to the experiment and consistent with the simulation models based on holetraps or the simpler structure as in Fig.10.

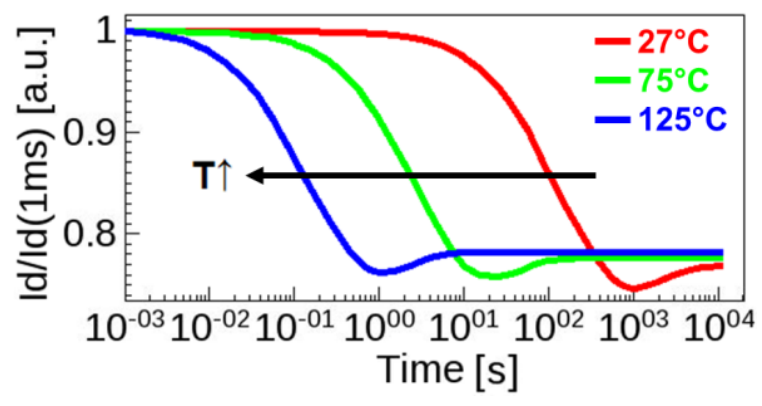

Fig.12: Simulated drain current transient of the model including the AlN and silicon layers, as illustrated in Fig. $11(\mathrm{~b})$, for $\mathrm{T}=27^{\circ} \mathrm{C}, 75^{\circ} \mathrm{C}$, $125^{\circ} \mathrm{C}$.

In this case, also the substrate current increases as a function of temperature, as reported in Fig. 13.

It has been discussed in the context of Fig. 8 that an increase in temperature weakens the capture process, therefore, by comparison, we conclude that (as shown in Fig.12) it is solely the substrate current and its dependence on the temperature that causes the decrease in drain current over time. This also implies that the extraction of trap properties becomes inaccurate if the physical mechanisms behind the vertical leakage are not 
taken into account and modelled properly.

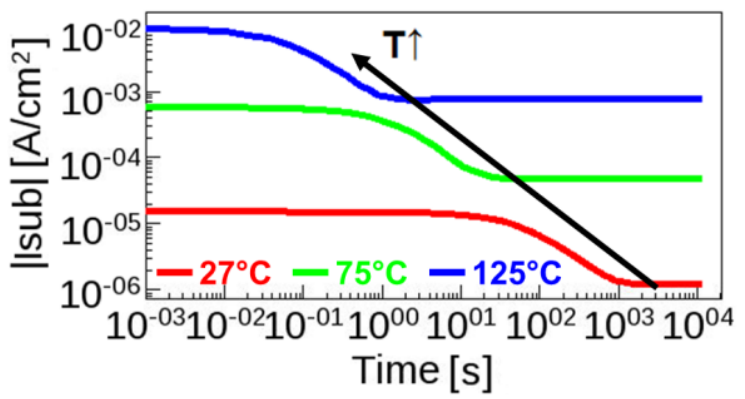

Fig.13: Simulated substrate current transient of the model including the AlN and silicon layers, as illustrated in Fig. $11(\mathrm{~b})$, for $\mathrm{T}=27^{\circ} \mathrm{C}, 75^{\circ} \mathrm{C}$, $125^{\circ} \mathrm{C}$.

Varying the barrier at the AlN-Si interface substantially modifies the leakage and, as a consequence, both the time constant and the magnitude of the drain current transient. This is shown in Fig.14. When the barrier is high, there is no decrease in current associated with electron traps, while as the barrier decreases the transient is faster and the decrease in current is more relevant.

To complete the analysis we have also evaluated the impact of varying the electron-trap energy on the drain current transient. This is reported in Fig. 15, where it is possible to note that moving the trap energy level from Ec- $0.65 \mathrm{eV}$ to Ec- $0.9 \mathrm{eV}$ has a minor effect on the time constant of the transient, while it has a relevant impact on the magnitude of the decrease in current.

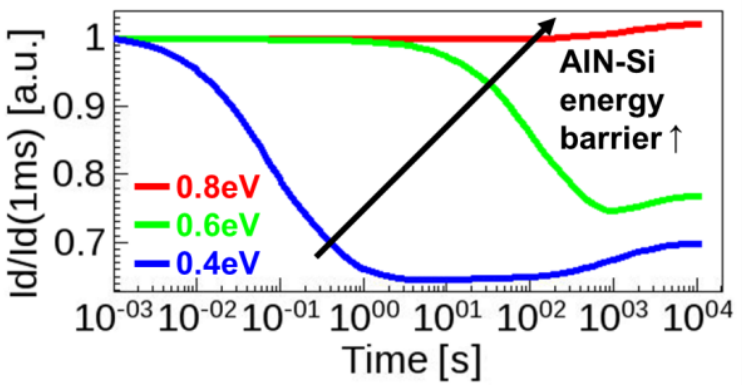

Fig.14: Simulated drain current transient as a function of the AlN-S energy barrier.

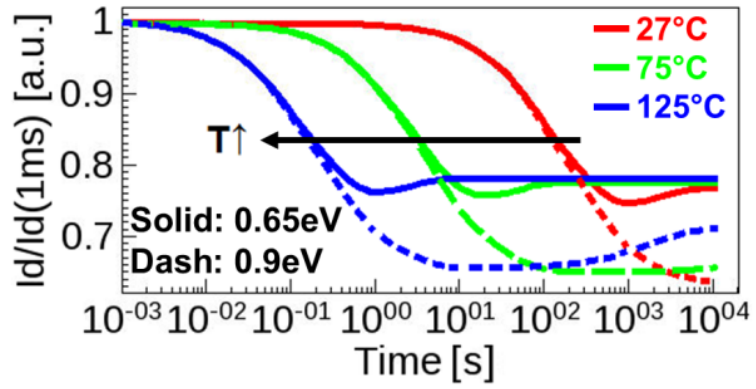

Fig.15: Comparison of drain current transient for $\mathrm{T}=27^{\circ} \mathrm{C}, 75^{\circ} \mathrm{C}$, $125^{\circ} \mathrm{C}$ when the electron-trap energy is changed from $0.65 \mathrm{eV}$ to $0.9 \mathrm{eV}$.

\section{Conclusions}

We have introduced an effective TCAD model for the study of the interaction between electrons injected from the substrate and buffer traps. We have clarified the impact of electron-traps in the buffer highlighting that increased vertical leakage above a certain level may lead to relevant Ron degradation and may explain inconsistencies observed when taking into account only hole-traps in the buffer.

However, below a certain leakage level $\left(10^{-6} \mathrm{~A} / \mathrm{cm}^{2}\right)$, the effect of the electron leakage from the substrate on the dynamic Ron is negligible.

In particular we have concluded that: (i) only high levels of vertical leakage currents due to electron injection from the substrate can affect the on-state strength of the 2DEG layer. Electrons injected from the substrate are trapped in the buffer and create an additional negative charge which results in lower 2DEG electron strength. In our simulations this effect is only present at leakage levels above $10^{-6} \mathrm{~A} / \mathrm{cm}^{2}$ and becomes of real concern above $10^{-5} \mathrm{~A} / \mathrm{cm}^{2}$.

(ii) while the stress bias used in our simulations has been fixed to $-100 \mathrm{~V}$, the conclusion that higher leakage leads to relevant trapping in electron traps may in principle be extended to higher voltages.

(iii) we have shown that the temperature dependence of the substrate and drain transient currents can be qualitatively reproduced and highlighted that various injection mechanisms must be taken into account to correctly analyse the trap properties.

(vi) in our experiments the vertical leakage current at $100 \mathrm{~V}$ is only $3 \mathrm{nA} / \mathrm{cm}^{2}$, therefore the decrease in the transient current in Fig. 1 cannot be directly attributed to electron trapping due to vertical leakage. Consistent to other studies, and also shown in Fig. 10, the decrease in the transient current is due to the hole emission from traps in the immediate vicinity of the 2DEG layer (below 
the 2DEG), due to the expansion of the electric field.

\section{References}

[1] Ishida et al., IEEE TED, 60(10), 3053-3059, (2013)

[2] Lyons et al., PHYS. REV. B 89, 035204 (2014)

[3] Uren et al. IEEE TED, 59(12), 3327-3333, (2012)

[4] Meneghini et al., IRPS 2015 (pp. 2E-2)

[5] Yang et al., ISPSD 2017 (pp. 101-104)

[6] Longobardi et al., ISPSD 2017, (pp. 227-230)

[7] Chini et al., IEEE TED, 63(9), 3473-3478, (2016)

[8] Cornigli et al., IEDM 2015 (pp. 5-3)

[9] Yacoub et al., IEEE TED, 64(3), 991-997, (2017) 